\title{
COUNTING MUONS TO PROBE THE NEUTRINO MASS SPECTRUM
}

\author{
CAROLINA LUJAN-PESCHARD ${ }^{1,2}$, GIULIA PAGLIAROLI ${ }^{1}$, FRANCESCO VISSANI $^{1,3}$ \\ ${ }^{1}$ INFN, LABORATORI NAZIONALI DEL GRAN SASSO, ASSERGI (AQ), ITALY \\ ${ }^{2}$ DEPARTAMENTO DE FISICA, DCEI, UNIVERSIDAD DE GUANAJUATO, LEÓN, GUANAJUATO, MÉXiCO \\ ${ }^{3}$ GRAN SASSO SCIENCE INSTITUTE (INFN), L'AQUILA, ITALY
}

\begin{abstract}
The experimental evidence that $\theta_{13}$ is large opens new opportunities to identify the neutrino mass spectrum. We outline a possibility to investigate this issue by means of conventional technology. The ideal setup turns out to be long baseline experiment: the muon neutrino beam, with $10^{20}$ protons on target, has an average energy of 6 (8) $\mathrm{GeV}$; the neutrinos, after propagating $6000(8000) \mathrm{km}$, are observed by a muon detector of 1 Mton and with a muon energy threshold of $2 \mathrm{GeV}$. The expected number of muon events is about 1000 , and the difference between the two neutrino spectra is sizeable, about $30 \%$. This allows the identification of the mass spectrum just counting muon tracks. The signal events are well characterized experimentally by their time and direction of arrival, and $2 / 3$ of them are in a region with little atmospheric neutrino background, namely, between $4 \mathrm{GeV}$ and $10 \mathrm{GeV}$. The distances from CERN to Baikal Lake and from Fermilab to KM3NET, or ANTARES, fit in the ideal range.
\end{abstract}

\section{INTRODUCTION}

The study of neutrinos from the sun [1], from the Earth's atmosphere [2] and from artificial sources [3] led to discover neutrino oscillations [4] proving the relevance of the matter effect [5, 6] for solar neutrinos. The most direct extension of the standard model requires 3 massive neutrinos. Such a picture, summarized following [7] in Fig. 1, permits us to account for most neutrino observations and to ask new questions; in particular, how to probe whether the neutrino spectrum is normal or inverted. After the experimental evidence that $\theta_{13}$ is large [8] a more precise formulation of this question is: how to take advantage of the matter effect in the Earth to solve this ambiguity.

Note that the experimental approaches that are presently implemented (or those planned before $\theta_{13}$ was known) have not been optimized to observe a large matter effect, and seem unable to guarantee very clear answers on which is the mass spectrum: see the recent nuTURN conference [9], where this issue has been debated and the conclusions of [10]. However, the most relevant energies are indicated directly by the theory: the matter effect is maximum when the matter and the vacuum terms, that describe oscillations, are comparable; this happens when the neutrino energies are in the range $5-10 \mathrm{GeV}$. Moreover, in order to have a large matter effect in the Earth, neutrinos have to cross a 


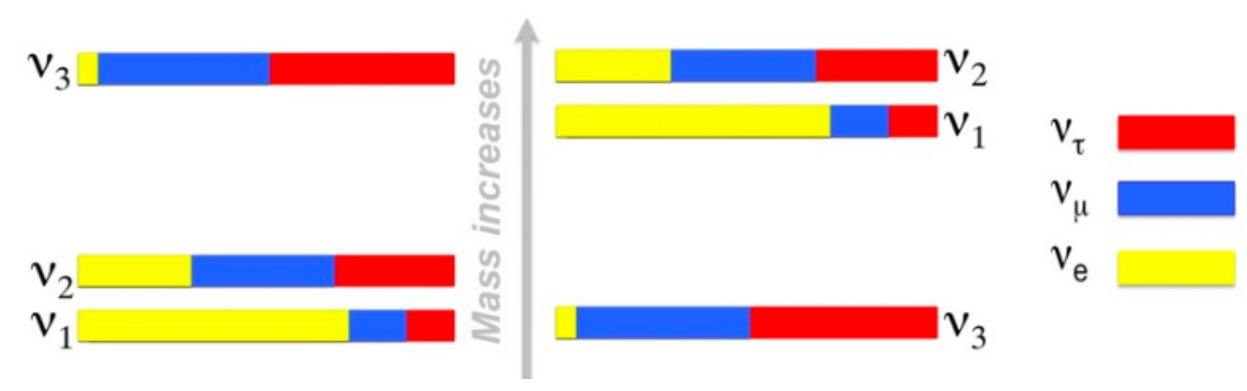

FiguRE 1. The two spectra that are compatible with present neutrino data: left, normal hierarchy; right, inverted hierarchy. The flavor content of each individual state with given mass, $\left|U_{\ell i}^{2}\right|$, is represented using the color code given in the rightmost part of the figure.

sizeable amount of matter, of the order of the Earth's radius, as discussed later on (Sect. 2) and in agreement with the 'oscillograms' of [11] or also with Fig. 3 of [12].

An idea to proceed is to try to identify an experimental sample of atmospheric neutrino events where the matter effect is large; in order to achieve this goal, muon neutrinos and antineutrinos have been considered, because the muons and the antimuons are supposed to be identifiable experimentally. Several specific options have been considered: e.g., a 50 kton magnetized iron detector, able to measure the charge of the muon [13] and to distinguish between neutrinos and antineutrinos; an argon detector of many 100 kton, able to measure also the energy of the hadrons scattered by the neutrinos, reconstructing better the neutrino energy [14]; a huge, $\sim 10$ Mton water Cherenkov muon detector [11, as a dense core of underwater/underice installations aimed at seeing high-energy neutrinos from cosmic sources. In any case, a very large detector mass is required, due to the need to probe relatively high energies, where the atmospheric neutrino flux is low. Furthermore, the use of atmospheric neutrinos implies certain limitations: 1) It is not possible to reconstruct the neutrino energies simply observing the muons, as in the largest water Cherenkov detectors, because a muon neutrino with energy $E$ will give a muon with energies from zero to this value. 2) If muons and antimuons cannot be distinguished, the impact of matter effect is diluted.

With these considerations in mind, we would like to suggest a different approach to emphasize the matter effect, insisting on the use of large (but relatively simple) muon detectors. We propose to send a muon neutrino beam produced in laboratory, maximizing the muon neutrino disappearance that is induced by the matter effect by a suitable choice of the distance of propagation and of the average energy of the beam $\mathbb{1}^{1}$ In the next section, we show that it is possible to arrange for a considerable amount of muon disappearance.

\footnotetext{
${ }^{1}$ Ref. [15, 16] have discussed electron neutrino appearance; however, the detection of a signal due to electrons is significantly more demanding than those due to muons. Ref. [17] discussed something closer to the present proposal; however, its authors have considered energy thresholds modeled on the existing neutrino telescopes, that are larger than our threshold: this has a significant impact on the ensuing conclusions.
} 

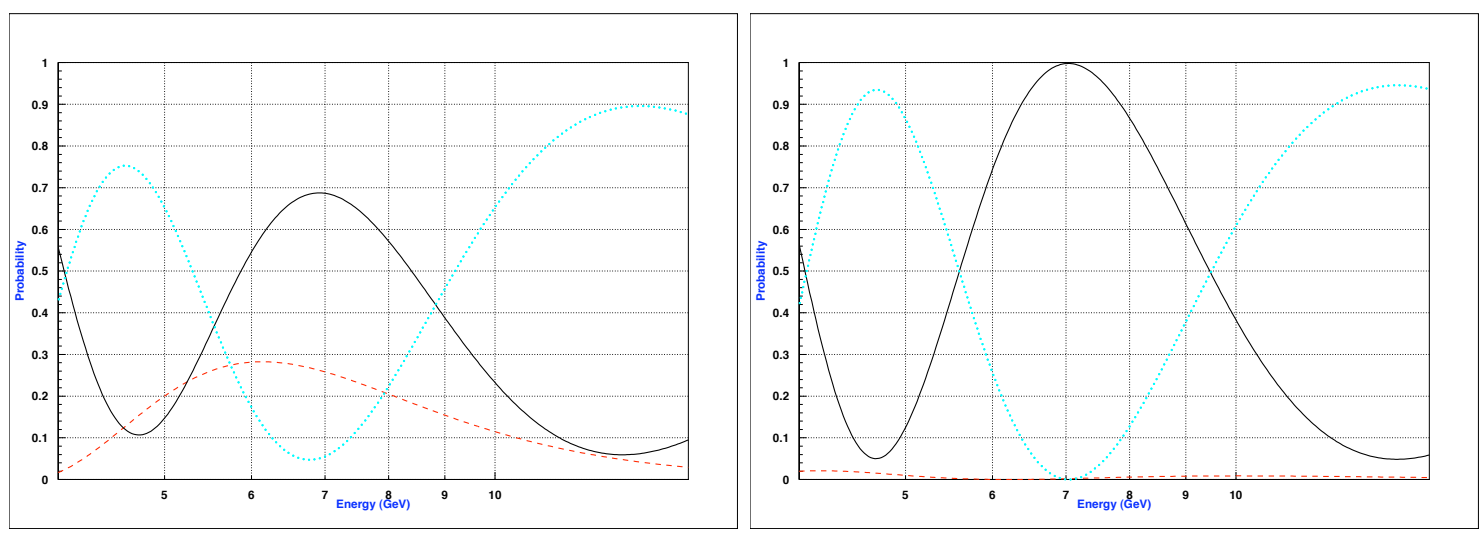

FiguRE 2. Oscillation probabilities for $L=7000 \mathrm{~km}$ : the continuous (black) line is $P_{\mu \mu}$; the dashed (red) line $P_{\mu e}$; the dotted (cyan) line $P_{\mu \tau}$. Left/right panel, normal/inverted hierarchy. The increase of $P_{\mu e}$ for normal hierarchy, due to matter effect, causes the decrease of $P_{\mu \mu}$.

Based on this remark, we argue that it is possible to achieve the goal of measuring the neutrino mass hierarchy with conventional technology, and moreover, by employing a wellknown type of neutrino beam and possibly taking advantage of sites that already host muon detectors. However, we need to improve the existing detectors, lowering their energy threshold to a few $\mathrm{GeV}$. In short, we argue that in order to distinguish normal from inverted hierarchy, we should simply count muons in the right type of experimental setup.

\section{MuON SURVIVAL PROBABILITy}

Let us begin the discussion from the case when only the largest $\Delta m^{2}$ matters. If $\theta_{13}$ is set to zero, we have 2-flavor vacuum oscillation: the muon survival probability oscillates as a function of the energy, its maxima correspond to the minima of $P_{\mu \tau}$ (and viceversa), and there is no difference between normal and inverted hierarchy. Then, let us consider the effect of $\theta_{13}$. Assuming normal hierarchy, $P_{\mu e}$ is amplified due to matter effect for certain energies. Therefore, around these energies, the muon survival probability,

$$
P_{\mu \mu}=1-P_{\mu e}-P_{\mu \tau}
$$

must decrease. Stated otherwise, we remark that the occurrence of electron neutrino appearance due to matter effect, i.e., an increase of $P_{\mu e}$, is strictly connected with the occurrence of muon neutrino disappearance, i.e., a decrease of $P_{\mu \mu}$, that is particularly remarkable when $P_{\mu \tau}$ is small. For inverted hierarchy, instead, $P_{\mu e}$ is suppressed by matter effect and the oscillations are very similar to the case when $\theta_{13}$ is set to zero.

Being interested in maximizing the difference between normal and inverted hierarchy, we consider the case when a local maximum of $P_{\mu \mu}$ is decreased as much as possible. This 
happens when the first minimum of $P_{\mu \tau}$, that drives the maximum of $P_{\mu \mu}$, falls close to the energy where $P_{\mu e}$ is large. Such a condition can be easily analyzed numerically, and we use the FORTRAN code developed for the study [14] where it is described. This code integrates numerically the full three flavor hamiltonian for neutrino oscillations in the Earth. The density of the Earth is described by the PREM model [18] and the code has a numerical accuracy of better than 1 part per million. Moreover, the code has been made publicly available in the web resource,

$$
\text { http://pcbat1.mi.infn.it/ battist/cgi-bin/oscil/index.r }
$$

Let us adopt the result of the global analysis of the Bari group [19], namely, $\theta_{12}=33.6^{\circ}$, $\theta_{13}=8.9 / 9.0^{\circ}, \theta_{23}=38.4 / 38.8^{\circ}, \delta=194 / 196^{\circ}, \Delta m_{12}^{2}=7.54 \times 10^{-5} \mathrm{eV}^{2}, \Delta m_{23}^{2}=$ $2.39 / 2.47 \times 10^{-3} \mathrm{eV}^{2}$; where the two values apply to normal/inverted hierarchy, respectively. For normal hierarchy we find that, when the neutrino energy $E$ and the corresponding oscillation length $L$ are about

$$
E=6,7,8 \mathrm{GeV} \text { and } L=6000,7000,8000 \mathrm{~km} \text { respectively }
$$

the maximum of $P_{\mu \mu}$ in which we are interested lowers to

$$
P_{\mu \mu}^{\max }(\mathrm{NH}) \sim 0.7
$$

The difference with $P_{\mu \mu}^{\max }(\mathrm{IH}) \sim 1$ (i.e., the case of inverted hierarchy) is as large as $30 \%$ for the distances $\sim R_{\oplus}$ of Eq. 2. As an example, we plot in Fig. 2 the case $L=7000 \mathrm{~km}$.

We verified that for other distances and/or for other energies, the effect diminishes, especially when $L$ decreases. Thus Eq. 2 identifies the optimal distances and energies to search for matter effect on $P_{\mu \mu}$, and impose conditions on the type of muon neutrino source and muon detectors needed for this purpose. We checked that these results do not depend much on the errors on most of the parameters, and in particular on CP-violating phase. For instance, consider the variation due to $\theta_{23}$; the present $3 \sigma$ range [19] implies that at $7800 \mathrm{~km} P_{\mu \mu}^{\max }(\mathrm{NH}) \sim 0.7_{+0.04}^{-0.15}$. This is not a very wide variation, and there are reasonable perspectives to improve $\theta_{23}$ in the next few years. Note however that if $\theta_{23}$ was larger than currently estimated, the effect could increase significantly. More discussion will be given later, in Sect. 5.1.

Appendix A offers some insight into the issue, thanks to suitable approximations and some simplified analytical descriptions of the matter effect. It also collects various relevant formulae; e.g., Eqs. 10 and 18 explain why the effect increases with $\sin ^{2} \theta_{23}$.

\section{Cross SECtion For NeUtrino-Muon production}

For the energies of interest, the leading interaction that yields observable muons is,

$$
\nu_{\mu}+N \rightarrow \mu^{-}+X
$$



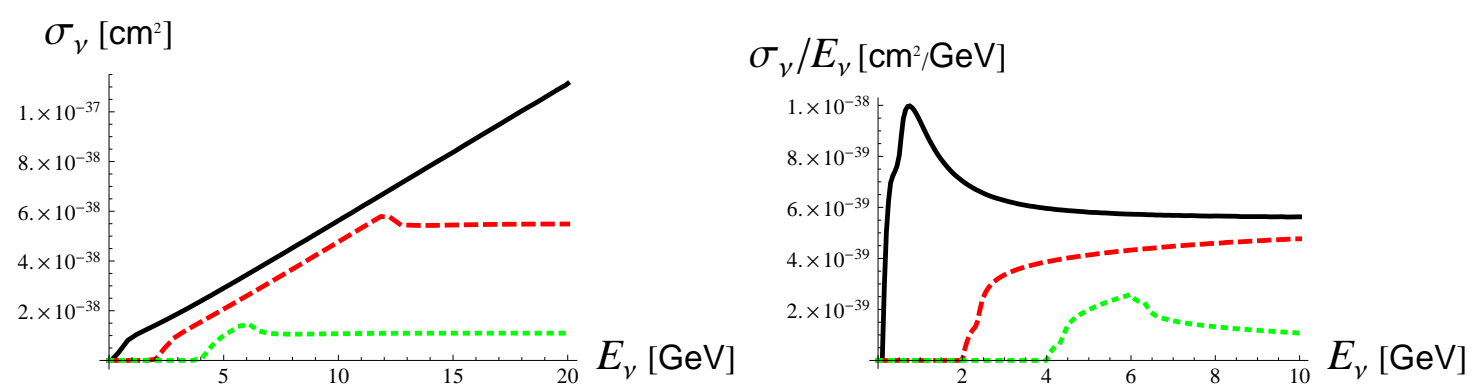

FIGURE 3. Various $\nu_{\mu} N \rightarrow \mu X$ cross sections as a function of the energy of the incoming $\nu_{\mu}$. The continuous (black) line is the total cross section; the dashed (red) line restricts the muon energy to $2<E_{\mu}<12 \mathrm{GeV}$; the dotted (green) to $4<E_{\mu}<6 \mathrm{GeV}$.

where $N$ is an average nucleon and $X$ is a set of hadrons; we consider water nuclei, therefore a neutron/proton ratio of $4 / 5$. We use the differential cross section $d \sigma / d E_{\mu}$ calculated in [20], following [21]. In view of the fact that the neutrino energies are not very large, we sum the following three contributions according to the hadronic mass of the final state $m_{X}^{2} \equiv p_{X}^{2}$ :

(1) The quasi-elastic contribution, with $M_{A}^{2}=0.95 \mathrm{GeV}^{2}$ and $F_{A}(0)=-1.26[22]$.

(2) The delta resonance, where we resort to CVC and PCAC and the parameterization of the form factors described in eqs. 12,13 and 18 of [23].

(3) The deep inelastic contribution for $m_{X}>1.4 \mathrm{GeV}$, with GRV94 partons [24].

We show in Fig. 3 the following integrated cross sections: 1) the total cross section; 2) the one where we integrate only the region of muon energy $2<E_{\mu}<12 \mathrm{GeV}$; 3) the same, restricted to the range $4<E_{\mu}<6 \mathrm{GeV}$. As customary after [21], also the ratio between the cross section and the neutrino energy is displayed $2^{2}$ Note that when $E_{\nu}$ is close to the threshold, the contribution of individual resonances considered (the nucleon and the delta) is clearly visible.

\section{NEEDS FOR THE EXPERIMENT}

4.1. Mass of the detector. The number of muon events scales with the mass of the detector $M_{\text {det }}$ and the distance $L$ as $N_{\mu} \propto M_{\text {det }} / L^{2}$. Thus, in order to have as many events as those in a detector of $10 \mathrm{kton}$ at $800 \mathrm{~km}$ from the source (roughly corresponding to the present generation long baseline experiment $\mathrm{NO} \nu \mathrm{A}$ [25]) at a distance of $8000 \mathrm{~km}$, we need a detector of about 1 Mton. Moreover, keeping in mind Fig. 2, it is easy to be convinced

\footnotetext{
2 At the energies in which we are interested, we have found an approximate scaling of the integrated cross section: $\sigma\left(E_{\nu}, E_{1}<E_{\mu}<E_{2}\right) \equiv \int_{E_{1}}^{E_{2}} \frac{d \sigma}{d E_{\mu}}\left(E_{\nu}, E_{\mu}\right) d E_{\mu} \approx f\left(E_{\nu}-E_{1}, E_{2}-E_{1}\right)$.
} 
that we need to detect muons from few to ten $\mathrm{GeV}$, which implies that we have to measure tracks of $10-40$ meters in water.

These requirements, concerning the mass of the detector and the length of the tracks to be revealed, point toward a large but relatively simple underwater or under-ice detector, that could resemble the PINGU [26] and ORCA [27] proposals that are being developed/considered by the IceCube and KM3NET collaborations, respectively. In the following, we will assume that the detector has a number of useful target nucleons of

$$
N_{\text {targ. }}=6 \times 10^{35}
$$

corresponding to a cube of water of $100 \mathrm{~m}$ in size, i.e., $M_{\mathrm{det}}=1 \mathrm{Mton}$, and leave a more detailed description of the detector for future work. Our results can be simply rescaled with the mass of the detector.

4.2. Source-detector distance. The next question is which arrangement of source and detector would fit the optimal range of distances identified above. Let us consider the existing neutrino lines [28] and a few sites of under-water and under-ice neutrino experiments. Their approximate coordinates in degrees are

$$
(\lambda, \phi)=\left\{\begin{array}{cl}
(+41.8,-88.3) & \text { Fermilab } \\
(+46.2,+6.0) & \text { CERN } \\
(+36.4,+140.6) & \text { J-PARC }
\end{array} \text { and }=\left\{\begin{array}{cl}
(-90,+0.0) & \text { South Pole } \\
(+36.3,+16.1) & \text { Sicily } \\
(+51.8,+104.3) & \text { Baikal Lake }
\end{array}\right.\right.
$$

'South Pole', 'Sicily' and 'Baikal Lake' correspond to the coordinates of IceCube, KM3NET and GVD, respectively. From the position versors $\vec{n}_{i}=\left(\cos \lambda_{i} \cos \phi_{i}, \cos \lambda_{i} \sin \phi_{i}, \sin \lambda_{i}\right)$ we find the relative distances as $L=R_{\oplus}\left|\vec{n}_{1}-\vec{n}_{2}\right|=R_{\oplus} \sqrt{2\left(1-\vec{n}_{1} \cdot \vec{n}_{2}\right)}$; or, using the web resource [29] one gets the minimum distance on the Earth surface $A$ (the arc) and thus $L=2 R_{\oplus} \sin \left[A /\left(2 R_{\oplus}\right)\right]$. We find the following distances, expressed in $\mathrm{km}$

\begin{tabular}{c|ccc} 
& Fermilab & CERN & J-PARC \\
\hline South Pole & 11600 & 11800 & 11400 \\
Sicily & $\mathbf{7 8 0 0}$ & 1230 & 9100 \\
Baikal Lake & 8700 & $\mathbf{6 3 0 0}$ & 3300
\end{tabular}

The pairs CERN to Baikal Lake and Fermilab to Sicily are at optimal distances. The first case would be somewhat more convenient, for its $1 / L^{2}$ factor is $50 \%$ larger. However, as we will show, also the second case offers reasonable opportunities for an experiment, and before continuing, we note that also the location of ANTARES, with $(\lambda, \phi)=(+42.8,+6.2)$, would be also appropriate, its distance from Fermilab being $6900 \mathrm{~km}$.

4.3. Properties of the neutrino beam. The existing neutrino beam from CERN has a mean energy twice higher than necessary to probe the matter effect, whereas it has already been shown that the NuMI neutrino beam of Fermilab can be arranged to fit our needs [30]. The estimated fluences, and more precisely those called HE and ME options [30], are evaluated for $10^{20}$ protons on target and at $1.04 \mathrm{~km}$ from the source. They 


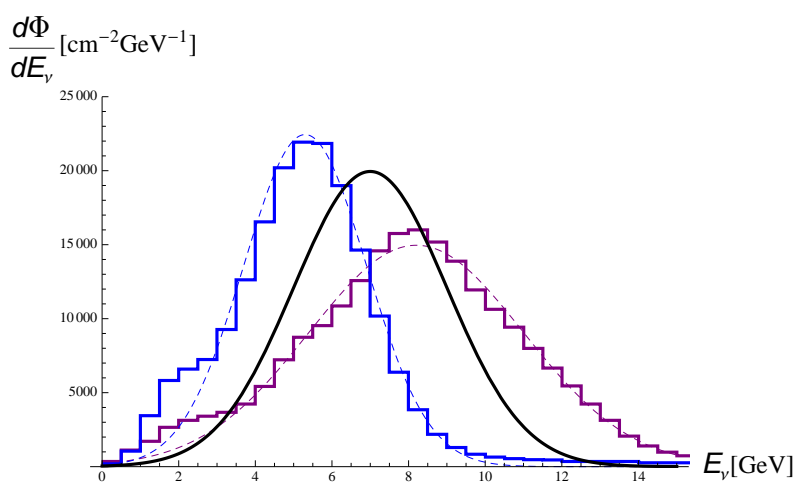

FiguRE 4. Differential neutrino fluences for a distance of $L=7800 \mathrm{~km}$. The two dashed gaussian distributions at the right and at the left are quite similar to the fluxes $\mathrm{ME}$ and HE of NuMI beam 30. for $10^{20}$ protons-on-target, shown as histograms. The black gaussian distribution with intermediate energies is assumed for the subsequent calculations. Compare with Fig. 2

can be reasonably approximated as gaussian distributions, proportional to $G(E, \bar{E}, \delta E)=$ $\exp \left[-(E-\bar{E})^{2} /\left(2 \delta E^{2}\right)\right] / \sqrt{2 \pi \delta E^{2}}$, with $\bar{E}=8.2$ (resp., 5.3) $\mathrm{GeV}$ and $\delta E=2.8$ (resp., 1.6) $\mathrm{GeV}$ as shown in Fig. 4, where we rescaled for a distance of $L=7800 \mathrm{~km}$. The fluence of a conventional muon neutrino beam, with properties intermediate between the above two cases (see Fig. 4) is approximated by,

$$
\frac{d \Phi}{d E}=\frac{10^{5} \nu_{\mu}}{\mathrm{cm}^{2}} \times G(E, \bar{E}, \delta E) \quad \text { with } \quad\left\{\begin{array}{c}
\bar{E}=7 \mathrm{GeV} \\
\delta E=2 \mathrm{GeV}
\end{array}\right.
$$

It corresponds to a beam of $10^{20}$ protons on target sent at a distance of $7800 \mathrm{~km}$. In the following, we will consider such a fluence for definiteness.

\section{Results}

The number of expected muon tracks contained in the detector, corresponding to muon energies larger than $E_{t h}$ and to a given fluence of muon neutrinos $d \Phi / d E$, is

$$
N_{\mu}=N_{\text {targ. }} \times \int_{E_{t h}} P_{\mu \mu}(E) \times \frac{d \Phi}{d E}(E) \times \sigma(E) d E
$$

When we use the number of targets as in Eq. 5, the fluence of Eq. 7 (see previous section) and the cross section to produce muons above $2 \mathrm{GeV}$ and below $12 \mathrm{GeV}$ (Sect. 3) we get

$$
N_{\mu}=\left\{\begin{array}{l}
950 \text { with normal hierarchy } \\
1300 \text { with inverted hierarchy }
\end{array}\right.
$$



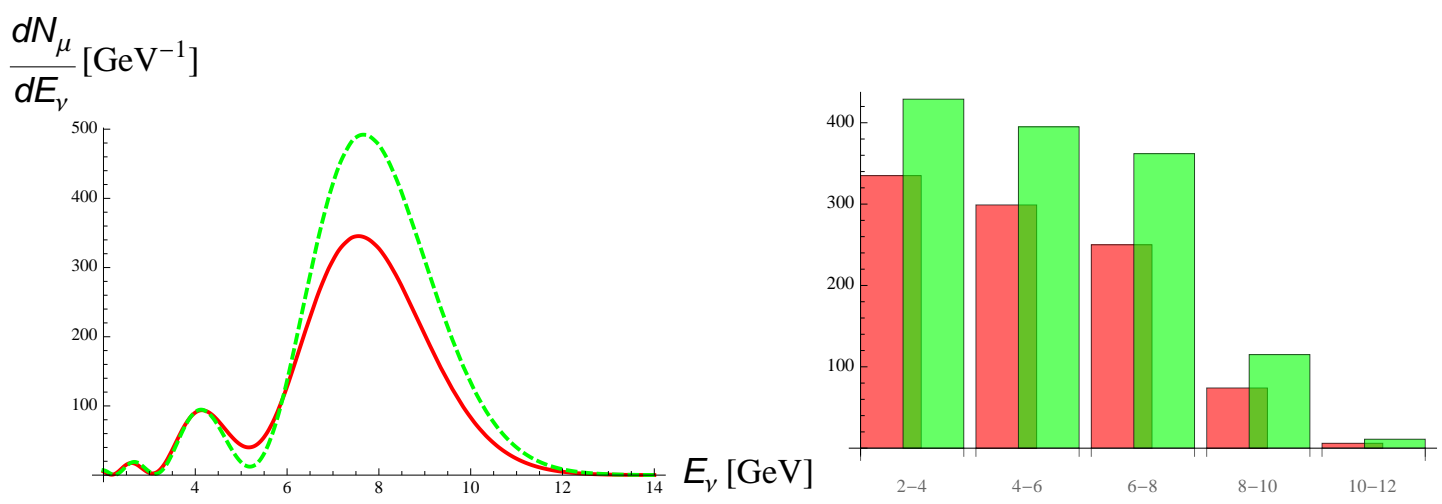

FIGURE 5. Left panel, distribution in the neutrino energy; the continuous curve (red) is for normal hierarchy, the dashed one (green) is for inverted hierarchy. Right panel, number of events in various windows of muon energy (2-4 GeV, 4-6 GeV, etc., as indicated below the abscissa) for normal hierarchy (left bars in the back, red) and for inverted hierarchy (right bars in front, green).

The difference is 30\%, as expected. The distribution of the events is shown in Fig. 5. Even if we retain only the events with muon energy above $4 \mathrm{GeV}$, in order to use a simpler detector or to avoid confusion with atmospheric neutrinos, we loose only $1 / 3$ of the events. The number of signals is ten times the expected statistical variance, $\sqrt{N_{\mu}}$, so that the difference between normal and inverted hierarchy cannot be mimicked by any plausible statistical fluctuation. The signal events can be distinguished by those from atmospheric neutrinos not only because of their spectra, but most of all, because the neutrinos arrive in bunches, due to the pulsed structure of the artificial neutrino beam, and to some extent also thanks to a certain degree of directionality at these energies-the angle between the neutrino and the produced muon being $\sim \sqrt{1 \mathrm{GeV} / E} \sim$ several tens of degrees.

It is also possible to cross check the results by using a muon anti neutrino beam (from $\pi^{-}$decay). In fact, the matter effect works in the opposite manner on antineutrinos, diminishing by $30 \%$ the number of $N_{\mu}$ for inverted hierarchy, rather than for normal hierarchy as for the neutrino beam considered above.

5.1. Stability of the results. In principle, various effects could affect the expectations, but none among those that we have considered is larger than few percent:

1) If the true oscillation parameters are not the present best-fit values, the number of expected muons will change. We find $N_{\mu}(\mathrm{NH})_{+3.4+2.7-2.1 \%}^{-3.5-3.0+0.9 \%}$ and $N_{\mu}(\mathrm{IH})_{+1.0+0.0-0.3 \%}^{-1.3+0.0-0.4 \%}$, where the superscript (resp., the subscript) percentage variations correspond to the upper (resp., lower) $1 \sigma$ value of $\theta_{23}, \theta_{13}$ and $\Delta m_{23}^{2}$ given in [19]; the variations with the other parameters 
are (much) smaller $3^{3}$ Thus, the expected changes are small. Note also that our knowledge of the oscillation parameters is likely to improve in the near future, diminishing this uncertainty further.

2) What would happen if some electron event contaminated the muon sample? In the range of energy between 2 and $12 \mathrm{GeV}$, we expect 390 electron-type events for normal hierarchy and less than 10 electron-type events for inverted hierarchy. Thus, it is enough that we reject $90 \%$ or more of this type of events, in order to have a contamination of the muon sample of $4 \%$ or less, that does not affect the result. (Note, incidentally, that if an electron sample can be clearly identified, this will be an additional signal that the matter effect is occurring on neutrinos. However, the electron signal depends quite crucially on the specific type of detector, and we prefer to stress the muon signal in this discussion, in view of its relative easiness of detection.).

3) Finally, we discuss the muon events caused by charged current interactions of tau neutrinos, followed by the decay $\tau \rightarrow \mu+\bar{\nu}_{\mu}+\nu_{\tau}$. The probability of oscillation $P_{\mu \tau}$ is large, but the beam is not optimized to see taus; moreover, the charged current cross section has a relatively high energy threshold. Due to these circumstances, and to the branching ratio $\mathrm{BR}=17.41 \%$, this reaction yields only 26 (resp., 24) muons for normal (resp., inverted) hierarchy with energies between 2 and $12 \mathrm{GeV}$. We conclude that the ensuing number of muon events is small. Moreover, the difference due to these additional muon events between the two hierarchies is negligible.

\section{Summary AND Discussion}

The existing experiments are compatible with two possible neutrino mass spectra: the normal hierarchy might resemble the spectra of the other fermions, depending on the mass of the lightest neutrino, but the inverted hierarchy surely does not. Presumably, a proof of inverted hierarchy would be as shocking and informative as the discovery of a sterile neutrino. The study of the matter effect in the Earth is the most direct way to identify the neutrino mass spectrum. Recall that the neutrino spectrum should be known to disentangle the effect of leptonic $\mathrm{CP}$ violation, and thus to measure the $\mathrm{CP}$ violating phase; moreover, the spectrum matters also for the interpretation of neutrinoless double beta decay, of cosmological search for neutrino masses, of supernova neutrinos, etc.

In this work, we have discussed a long baseline neutrino experiment, aimed to study the matter effect in the Earth, by taking advantage of a large muon disappearance. We have argued that the ideal conditions are obtained for muon neutrinos with average energies of 6 (8) $\mathrm{GeV}$, that propagate for a distance of $L=6000(8000) \mathrm{km}$. A previous similar

\footnotetext{
3 The smallness of the variation due to $\Delta m_{23}^{2}$ is due to the selected neutrino beam: indeed, the signal has been almost maximized with the present best-fit values of the oscillation parameters.

${ }^{4}$ The non-optimal beam energy gives a factor $\sim 1 / 2$; the tau-production threshold, along with the fact that part of the energy is carried away by the two neutrinos in the final state, gives a factor $\sim 1 / 3$. These factors, along with the BR, explain the difference with the $\sim 1000$ muon signal discussed above.
} 
proposal, [17, discussed larger detectors with higher energy thresholds modeled on neutrino telescopes; other apparently related proposals [15, 16] considered electron neutrino detection instead, that is significantly more demanding than muon detection.

We have shown that a conventional (water Cherenkov) muon detector of 1 Mton along with a conventional muon neutrino beam $\left(10^{20}\right.$ protons on target) can observe $\sim 1000$ signal events, even at a relatively large distance of $7800 \mathrm{~km}$. The signal is composed by muons between $2-10 \mathrm{GeV}$, well characterized experimentally, and the muons can be identified as $10-40 \mathrm{~m}$ long tracks; moreover, $2 / 3$ of them are above $4 \mathrm{GeV}$. Due to the matter effect, the inverted hierarchy yields $30 \%$ more events than the normal hierarchy case. Thus, the difference induced by the matter effect is quite large and even a moderate understanding of the artificial neutrino beam should suffice to identify experimentally which is the neutrino mass spectrum.

\section{ACKNOWLEDGMENTS}

We thank G. Battistoni, A. Capone, R. Coniglione, P. Coyle, G.V. Domogatsky, S. Galatà, M. Goodman, P. Lipari, S. Ragazzi, G. Riccobene, F. Terranova, A. Varaschin, L. Votano and an anonymous Referee of EPJC for useful discussions. FV is grateful to the Organizers and the Participants in the Orca meeting at Paris for precious feedback [31].

\section{Appendix A. REMARKs on the MATter EFfECT}

In this appendix, we examine the oscillations by employing some simplifying assumptions, in order to obtain a qualitative understanding of the results in Sect. 2; we consider oscillations with a single scale, and we also consider oscillations in constant matter density. In fact, the second hypothesis is rather inaccurate in the conditions in which we are interested, and we need (and we use) a more accurate evaluation of the oscillation probabilities for the actual calculations. However, a qualitative discussion based on simple-minded analytical results complements usefully the numerical results discussed in the main text.

We try to go immediately to the main point, postponing derivations and refinements. Under suitable assumptions the probability that a muon converts into an electron is simply,

$$
P_{\mu e}=\sin ^{2} \theta_{23} \sin ^{2} 2 \widetilde{\theta_{13}} \sin ^{2} \widetilde{\varphi} \text { with } \widetilde{\varphi}=\frac{\widetilde{\Delta m^{2}} L}{4 E}
$$

Most of the results in which we are interested follow from the above simple formula. In Eq. 10, we introduced the usual matter-modified mixing angle and squared-mass-difference

$$
\left\{\begin{array}{l}
\sin 2 \widetilde{\theta_{13}}=\sin 2 \theta_{13} / \Delta \\
\cos 2 \widetilde{\theta_{13}}=\left(\cos 2 \theta_{13}-\varepsilon\right) / \Delta \quad \text { where } \Delta= \pm \sqrt{\left(\cos 2 \theta_{13}-\varepsilon\right)^{2}+\sin ^{2} 2 \theta_{13}} \\
\widetilde{\Delta m^{2}}=\Delta m^{2} \times \Delta
\end{array}\right.
$$


The sign of $\Delta$ is matter of convention; the ratio between matter and vacuum term is,

$$
\varepsilon \equiv \pm \frac{\sqrt{2} G_{F} n_{e}}{\Delta m^{2} /(2 E)} \approx \pm \frac{\rho}{5.5 \mathrm{~g} / \mathrm{cm}^{3}} \times \frac{Y_{e}}{1 / 2} \times \frac{2.4 \times 10^{-3} \mathrm{eV}^{2}}{\Delta m^{2}} \times \frac{E}{5.5 \mathrm{GeV}}
$$

where $G_{F}$ is the Fermi coupling and we identify $\Delta m^{2}$ with $\Delta m_{23}^{2}$. Now, instead, the sign is important: it is plus for normal hierarchy and minus for inverted hierarchy. Considering the average matter density of the Earth $\rho=5.5 \mathrm{~g} / \mathrm{cm}^{3}$ and $Y_{e}=1 / 2$, we get $n_{e}=1.7 \times 10^{24}$ $\mathrm{e}^{-} / \mathrm{cm}^{3}$ for the electronic density. Thus, the characteristic length of MSW theory is,

$$
L_{*} \equiv \frac{1}{\sqrt{2} G_{F} n_{e}} \sim 1000 \mathrm{~km}
$$

We see that, for normal hierarchy, the maximum of $P_{\mu e}$ obtains when: (1) $\Delta$ is as small as possible, in order to maximize $\sin 2 \widetilde{\theta_{13}}$; moreover, $(2)$ the phase of propagation is $\widetilde{\varphi} \sim \pi / 2$. These conditions are met when the neutrino energy and the propagation distance are,

$$
E_{\max }=\frac{\Delta m^{2} L_{*}}{2} \cos 2 \theta_{13} \sim 5.5 \mathrm{GeV} \text { and } L_{\max }=\frac{\pi L_{*}}{\tan 2 \theta_{13}} \sim 9000 \mathrm{~km}
$$

In the case of inverted hierarchy, the matter effect depresses $P_{\mu e}$, that becomes negligible.

In principle, one could check this simple prediction concerning $P_{\mu e}$, however it is practically easier to study muons rather than electrons. Then, let us consider the survival probability $P_{\mu \mu}$, focussing again on the normal hierarchy case. We want that a local maximum of $P_{\mu \mu}$, resulting from $P_{\mu \tau}$ and from $P_{\mu e}$, is as small as possible. Thus, we are interested in the case when the minimum of $P_{\mu \tau}$ happens in the vicinity of the energy identified in Eq. 14. When the phase of oscillation of $P_{\mu \tau}$ is close to the vacuum phase, the condition $\Delta m^{2} L /\left(2 E_{\max }\right)=2 \pi$ gives $L \sim 6000 \mathrm{~km}$. This suggests that the distance that amplifies the matter effect on $P_{\mu \mu}$ is between 6000 and $9000 \mathrm{~km}$, that does not disagree severely with the quantitative conclusions of the precise numerical analysis of Sect. 2.

Finally, we collect more arguments and technical remarks concerning the matter effect. Let us write in full generality the amplitude of three flavor neutrino oscillations

$$
\mathcal{A}=\operatorname{Texp}\left[-i \int d t \mathcal{H}_{\nu}(t)\right]=R_{23} R_{\delta}\left(\begin{array}{ccc}
a_{11} & a_{12} & a_{13} \\
a_{21} & a_{22} & a_{23} \\
a_{31} & a_{32} & a_{33}
\end{array}\right) R_{\delta}^{*} R_{23}^{t}
$$

where $a_{i j}$ depend upon $\Delta m_{23}^{2}, \theta_{13}, \Delta m_{12}^{2}, \theta_{12}$, and $H= \pm 1$ (the type of mass hierarchy, normal/inverted): see in particular Eqs. 1, 3, 5, 6 of [14]. By solving numerically the evolution equations, we calculate the complex numbers $a_{i j}$ and therefore the amplitudes and the probabilities. At this level, there is no approximation (except the numerical ones).

When the "solar" $\Delta m_{12}^{2}$ is set to zero-i.e., when its effects are negligible-the only nonzero out-of-diagonal elements $a_{i j}$ in Eq. 15 are $a_{13}$ and $a_{31}$. The CP violating phase $\delta$ drops out from the probabilities $P_{\ell \ell^{\prime}}=\left|\mathcal{A}_{\ell^{\prime} \ell}\right|^{2}$, that moreover become symmetric, $P_{\ell \ell^{\prime}}=P_{\ell^{\prime} \ell}$ for 
each $\ell, \ell^{\prime}=e, \mu, \tau$. Therefore, in this approximation we have 3 independent probabilities and all the other ones are fixed. We can chose, e.g.,

$$
P_{e \mu}=\sin ^{2} \theta_{23}\left|a_{13}\right|^{2}, P_{e \tau}=\cos ^{2} \theta_{23}\left|a_{13}\right|^{2}, P_{\mu \tau}=\sin ^{2} \theta_{23} \cos ^{2} \theta_{23}\left|a_{33}-a_{22}\right|^{2},
$$

so that, e.g., $P_{e e}=1-P_{\mu e}-P_{\tau e}=\left|a_{11}\right|^{2}$. From these formulae we obtain

$$
P_{\mu e}=\sin ^{2} \theta_{23}\left(1-P_{e e}\right) \text { and } P_{\mu \tau}=\frac{1}{4} \sin ^{2} 2 \theta_{23}\left|1-\sqrt{P_{e e}} e^{i \hat{\varphi}}\right|^{2},
$$

where $\hat{\varphi}$ is a (rapidly varying) phase factor. Two important remarks are in order:

(1) The last equation shows that $P_{\mu e}$ is large in the region where $P_{e e}$ is small, and that $P_{\mu \tau}$ remains close to zero in the first non-trivial minimum near $\hat{\varphi}=2 \pi$, even when $P_{e e} \approx 0.3-0.4$ due to matter effect.

(2) The sign of $\Delta m^{2}$ controls the sign of the vacuum hamiltonian; therefore, switching between the two mass hierarchies or switching between neutrinos and antineutrinos has the same effect; e.g., $P_{e \mu}(\mathrm{IH})=P_{\bar{e} \bar{\mu}}(\mathrm{NH})$.

The first remark is consistent with our numerical findings, that $P_{\mu e}$ is amplified and $P_{\mu \tau}$ does not deviate strongly from its behavior in vacuum in the conditions that are relevant for our discussion.

Proceeding further with the approximations, and considering at this point the case of constant matter density, we obtain simple and closed expressions. For the case of normal mass hierarchy, they read:

$$
\begin{aligned}
& a_{13}=a_{31}=-i \sin \widetilde{\varphi} \sin 2 \widetilde{\theta_{13}} \\
& a_{11}=\cos \widetilde{\varphi}+i \sin \widetilde{\varphi} \cos 2 \widetilde{\theta_{13}}=a_{33}^{*} \\
& a_{22}=\cos \widetilde{\varphi}^{\prime}+i \sin \widetilde{\varphi}^{\prime}
\end{aligned}
$$

where

$$
\widetilde{\varphi}^{\prime}=\frac{\Delta m^{2} L}{4 E}(1+\varepsilon)
$$

From Eqs. 16 and 18, we recover the expression of Eq. 10, used in the above discussion. In the approximation of constant matter density, the phase $\hat{\varphi}$ entering the expression of the probability $P_{\mu \tau}$ is given by $\sqrt{P_{e e}} \cos \hat{\varphi} \equiv \cos \widetilde{\varphi} \cos \widetilde{\varphi}^{\prime}-\sin \widetilde{\varphi} \sin \widetilde{\varphi}^{\prime} \cos 2 \widetilde{\theta_{13}}$. This is close to the vacuum phase when $\varepsilon$ is large or small in comparison to 1 : in fact, we have $\cos 2 \widetilde{\theta_{13}} \sim \pm 1$ and $\widetilde{\varphi} \sim \pm \Delta m^{2} L /(4 E)(1-\varepsilon)$ from Eq. 11 , so that $\cos \hat{\varphi} \sim \cos \left[\Delta m^{2} L /(2 E)\right]$. 


\section{REFERENCES}

[1] B.T. Cleveland et al., Astrophys. J. 496 (1998) 505. K.S. Hirata et al. [Kamiokande-II Coll.], Phys. Rev. D 44 (1991) 2241 [E-ibid. D 45 (1992) 2170]. S. Fukuda et al. [Super-Kamiokande Coll.], Phys. Rev. Lett. 86 (2001) 5651. W. Hampel et al. [GALLEX Coll.], Phys. Lett. B 447 (1999) 127. J.N. Abdurashitov et al. [SAGE Coll.], Phys. Rev. C 60 (1999) 055801. M. Altmann et al. [GNO Coll.], Phys. Lett. B 616 (2005) 174. Q.R. Ahmad et al. [SNO Coll.], Phys. Rev. Lett. 89 (2002) 011301. C. Arpesella et al. [Borexino Coll.], Phys. Rev. Lett. 101 (2008) 091302.

[2] Y. Fukuda et al. [Kamiokande Coll.], Phys. Lett. B 335 (1994) 237. Y. Fukuda et al. [Super-Kamiokande Coll.], Phys. Rev. Lett. 81 (1998) 1562. M. Ambrosio et al. [MACRO Coll.], Phys. Lett. B 434 (1998) 451. W.W.M. Allison et al. [Soudan-2 Coll.], Phys. Lett. B 449 (1999) 137.

[3] M.H. Ahn et al. [K2K Coll.], Phys. Rev. Lett. 90 (2003) 041801. D.G. Michael et al. [MINOS Coll.], Phys. Rev. Lett. 97 (2006) 191801. S. Abe et al. [KamLAND Coll.], Phys. Rev. Lett. 100 (2008) 221803. N. Agafonova et al. [OPERA Coll.], Phys. Lett. B 691 (2010) 138. K. Abe et al. [T2K Coll.], Phys. Rev. D 85 (2012) 031103.

[4] B. Pontecorvo, Sov. Phys. JETP 6 (1957) 429 and 26 (1968) 984; Z. Maki, M. Nakagawa and S. Sakata, Prog. Theor. Phys. 28 (1962) 870.

[5] L. Wolfenstein, Phys. Rev. D 17 (1978) 2369. S.P. Mikheev and A.Yu. Smirnov, Sov. J. Nucl. Phys. 42 (1985) 913 [Yad. Fiz. 42 (1985) 1441].

[6] V. D. Barger, K. Whisnant, S. Pakvasa and R. J. N. Phillips, Phys. Rev. D 22 (1980) 2718. P. Langacker, J. P. Leveille and J. Sheiman, Phys. Rev. D 27 (1983) 1228. G. V. Dass and K. V. L. Sarma, Phys. Rev. D 30 (1984) 80.

[7] A.Yu. Smirnov, "Neutrino Masses and Mixing," hep-ph/9611465, presented at the 28 International Conference on High-Energy Physics (ICHEP 96), Warsaw, Poland.

[8] G.L. Fogli, E. Lisi, A. Marrone, A. Palazzo and A.M. Rotunno, Phys. Rev. Lett. 101 (2008) 141801; K. Abe et al. [T2K Coll.], Phys. Rev. Lett. 107 (2011) 041801 and arXiv:1304.0841; F.P. An et al. [DAYA-BAY Coll.], Phys. Rev. Lett. 108 (2012) 171803; J.K. Ahn et al. [RENO Coll.], Phys. Rev. Lett. 108 (2012) 191802; Y. Abe et al. [Double Chooz Coll.], Phys. Rev. D 86 (2012) 052008.

[9] See discussion at nuTURN conference, web site http://nuturn2012.lngs.infn.it/

[10] M. Blennow, T. Schwetz, JHEP 1208 (2012) 058 [E.-ibid. 1211 (2012) 098]; A. Ghosh, T. Thakore, S. Choubey, arXiv:1212.1305 [hep-ph].

[11] E.K. Akhmedov, S. Razzaque and A.Yu. Smirnov, arXiv:1205.7071 [hep-ph].

[12] B. Bajc, F. Nesti, G. Senjanovic and F. Vissani, "Perspectives in neutrino physics." Frascati Physics Series, Vol. 30 (2003) page 103-143. Proceedings of $17^{t h}$ La Thuile conference, ed. M. Greco.

[13] A. Geiser [MONOLITH Coll.], Nucl. Instrum. Meth. A 472 (2000) 464; T. Tabarelli de Fatis, Eur. Phys. J. C 24 (2002) 43; D. Indumathi [INO Coll.], Pramana 63 (2004) 1283; N.K. Mondal [INO Coll.], Pramana 79 (2012) 1003.

[14] G. Battistoni, A. Ferrari, C. Rubbia, P.R. Sala, F. Vissani, "Atmospheric neutrinos in a large liquid argon detector," hep-ph/0604182.

[15] J. Tang and W. Winter, JHEP 1202 (2012) 028 arXiv:1110.5908 [hep-ph]].

[16] S. K. Agarwalla and P. Hernandez, JHEP 1210 (2012) 086 [arXiv:1204.4217 [hep-ph]].

[17] K. Dick, M. Freund, P. Huber and M. Lindner, Nucl. Phys. B 588 (2000) 101 hep-ph/0006090.

[18] A. M. Dziewonski and D. L. Anderson, Phys. Earth Planet. Interiors 25 (1981) 297.

[19] G.L. Fogli et al., Phys. Rev. D 86 (2012) 013012.

[20] S.R. Dugad and F. Vissani, Phys. Lett. B 469 (1999) 171.

[21] P. Lipari, M. Lusignoli, F. Sartogo, Phys. Rev. Lett. 74 (1995) 4384.

[22] C.H. Llewellyn Smith, Phys. Rept. 3 (1972) 261.

[23] L. Alvarez-Ruso, S.K. Singh, M.J. Vicente Vacas, Phys. Rev. C57 (1998) 2693.

[24] M. Glück, E. Reya and A. Vogt, Zeit. Phys. C67 (1995) 433. 
[25] D.S. Ayres et al. [NO $\nu \mathrm{A}$ Coll.], "NO $\nu$ A: Proposal to build a 30 kiloton off-axis detector to study $\nu_{\mu} \rightarrow \nu_{e}$ oscillations in the NuMI beamline," hep-ex/0503053. Web site: http://www-fnal.nova.gov

[26] D.J. Koskinen, Mod. Phys. Lett. A 26 (2011) 2899.

[27] http://agenda.infn.it/conferenceDisplay ·py?conf Id=5510

[28] J. Beringer et al. (Particle Data Group), Phys. Rev. D86 (2012) 010001, Sect.29.

[29] http://www.daftlogic.com/projects-google-maps-distance-calculator.htm

[30] S. Kopp, M. Bishai, M. Dierckxsens, M. Diwan, A.R. Erwin, D.A. Harris, D. Indurthy and R. Keisler et al., Nucl. Instrum. Meth. A 568 (2006) 503.

[31] https://indico.in2p3.fr/conferenceOtherViews. py?view=standard\&conf Id=8261 